\title{
Aconite Poisoning with Good Outcome, Case Study and Literature Review
}

\author{
Abdulrahman Alalmay', Ali Al Bashabshe', Nouf Al Shamrani' ${ }^{2}$ Lujain Bin Othman'2, \\ Musa Alfaifi'2, Yahya Algarni' ${ }^{2}$ Ahmed Awwadh², Ali Alhanash', Hassan Ogran1, \\ Khalid Sinnah ${ }^{2}$, Mostafa El Razzaz ${ }^{3}$ \\ ${ }^{1}$ King Khaled University, Abha, KSA \\ ${ }^{2}$ Ministry of Health, Abha, KSA \\ ${ }^{3}$ Internal Medicine, Ain Shams University, Cairo, Egypt \\ Email:Dr.almay@yahoo.com, albshabshe@yahoo.com,dr.nouf2022@hotmail.com,lu-jain.med@live.com,Alfaifim@gmail.com, \\ Dr.YaGarni@Gmail.com,M3lwee@gmail.com, Ali.m.alhanash@gmail.com, hassanogran@gmail.com, khalidsanh@gmail.com, \\ dr.mostafa_elrazzaz@med.asu.edu.eg
}

How to cite this paper: Alalmay, A., Al Bashabshe, A., Al Shamrani, N., Othman, L.B., Alfaifi, M., Algarni, Y., Awwadh, A., Alhanash, A., Ogran, H., Sinnah, K. and El Razzaz, M. (2020) Aconite Poisoning with Good Outcome, Case Study and Literature Review. Case Reports in Clinical Medicine, 9, 217-222.

https://doi.org/10.4236/crcm.2020.98030

Received: June 26, 2020

Accepted: August 3, 2020

Published: August 6, 2020

Copyright $\odot 2020$ by author(s) and Scientific Research Publishing Inc. This work is licensed under the Creative Commons Attribution International License (CC BY 4.0).

http://creativecommons.org/licenses/by/4.0/

\begin{abstract}
Aconite poisoning has been reported in previously published literature and case reports. It may occur due to accidental consumption of aconite tubers or when it is used in medicinal preparations. In this case report we describe a case of aconite poisoning in a primary case of anxiety disorder, after use of herbal medicine. He gave history of consumption of herbal remedy with honey from folk medicine with unknown content. Our patient presented with symptoms of vomiting, diarrhea, numbness and dizziness that are common symptoms of aconite poisoning. The management was mainly supportive care depending on the symptoms. Our patient recovered after 30 hours, probably due to slow elimination of the metabolites overtime. Our case represents one case where poisoning was not due to wrong consumption but due to drug-drug interaction. It is important that physicians should take drug interactions into consideration while prescribing drugs and also educate patients regarding the same.
\end{abstract}

\section{Keywords}

Aconite, Herbal Medications, Poisoning, Anxiety Disorder

\section{Introduction}

Traditional or herbal medications have become an essential part of alternative 
medicine. Although the common perception for taking such medications is usually to avoid adverse effects of allopathic medicine, there is a lack of safety information. With an increase in the use of herbal medications, an increase in reporting of adverse events has been observed [1]. These adverse events have been observed due to various reasons like quality issues, poor quality herbal material, incorrect or misidentified herbs, incorrect processing methods, supply of adulterated, or contaminated herbs or product [2]. The attributing factor in this is poor regulation of manufacture and sale of herbal products. Also, patients usually self-medicate on these products which can also be dangerous.

Aconite poisoning has been reported in previously published literature and case reports. It may occur due to the accidental consumption of aconite tubers or when it is used in medicinal preparations. In Asia, aconite roots are commonly used in traditional or herbal medicine as analgesic, anti-inflammatory, and cardiotonic agents [3]. Although these are properly processed to reduce the amounts of toxic alkaloids, aconite poisoning has been reported with the use of these preparations. The management is mainly supportive with monitoring of vital parameters. Inotropic therapy may be used to overcome hypertension and atropine for bradycardia.

In this case report, after obtaining the verbal consent of the patient we describe a case of aconite poisoning in a primary case of anxiety disorder, after the use of herbal medicine.

\section{Case Report}

\subsection{Complains and Clinical History}

A 27 years old, male patient presented to the emergency room on $5^{\text {th }}$ March 2020 , at $5 \mathrm{pm}$ with complains of dizziness and vomiting for past one hour. $\mathrm{He}$ gave history of consumption of herbal remedy with honey from folk medicine. The content of this herbal remedy was not known. After an hour of using it, he developed tingling sensation in the tongue and blurry vision and perioral numbness. Soon he started feeling dizziness. The patient also complained of vomiting and diarrhea. Vomiting more than 8 episodes and had mainly undigested food while he had 3 episodes of diarrhea.

There were no complains of abdominal pain, constipation, shortness of breath, chest pain, orthopnea. No change in urine color, amount, odor or frequency was seen.

\subsection{Past History}

The patient was a known case of anxiety disorder and was on fluoxetine $20 \mathrm{mg}$ once daily and olanzapine $2.5 \mathrm{mg}$ once daily. The patient didn't have any past history of allergy to food or drug. Vaccination history was complete, according to Saudi Vaccination schedule. There was no history of recent blood transfusion. No any significant past surgical history. He is living in a family house with an average income. He is on regular family diet. 


\subsection{Clinical Examination}

The patient was conscious, alerted and well-oriented. On examination, he was hypotensive with blood pressure of $92 / 43 \mathrm{~mm}$ of $\mathrm{Hg}$ and a mean arterial blood pressure of 59. Pulse rate was 58 beats per minute with a regular rhythm. Heart sounds were normal with no added sound. Chest was clear with a respiratory rate of 25 per minute.

Abdomen was soft and lax with no tenderness or organomegaly on palpation. Scar mark of appendectomy was present. No focal neurological deficit was seen.

\subsection{Toxicological Findings}

The Aconitum alkaloids can be extracted from the serum, urine and even vomit. After extraction and purification procedures of Aconiturn alkaloids, they were quantitated using Multiple Reaction Monitoring chromatography. Figure 1 shows the findings from measurements of the samples. The main alkaloid observed among all samples was Aconitine with an intensity of almost $6600 \mathrm{cps}$; Methylyaconitine, Mesaconitine, and Hypaconitine were also detected (Figure 1).

After seeing these symptoms and signs, we suspected a poisoning case. After collecting and amount of samples brought to emergency department and send later to toxicology lab, the examination showed aconite substance along with other substances.

\pm Aconitine (Mass/FragMass/RT//lsotope/Library/Formula/lon Ratio)

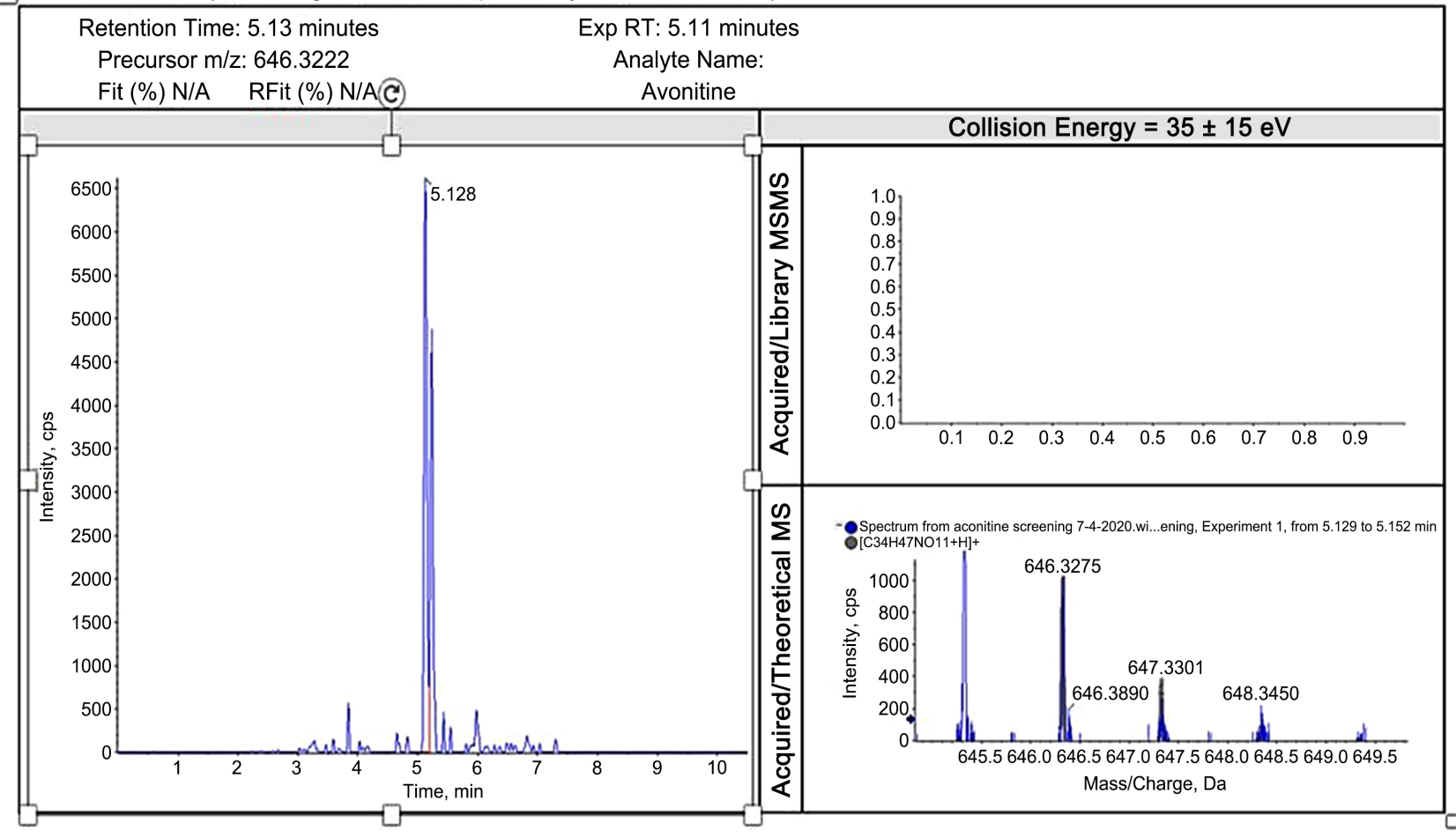

Figure 1. MRM chromatograms detecting Aconitine concentration in the sample (spike). 


\subsection{Management}

The management of this patient was mainly supportive care. Atropine $0.5 \mathrm{mg}$ was intravenous administrated as long as the heart rate was below 45 beats per minutes. To manage hypotension, 2-liters of normal saline were started along with epinephrine $0.5 \mathrm{mcg}$. For bradycardia, atropine $0.5 \mathrm{mg}$ was given for two times after cardiology consultation.

There was no need for a lavage given that the patient had more than 8 episodes of vomiting in addition to episodes of diarrhea.

Hypotension and bradycardia kept fluctuating for up to 28 hours after admission. Soon after it started it remained stable, with blood pressure of ... and heart rate of ...

\subsection{Recovery/Follow-Up}

One week after, the patient returned to the outpatient department (OPD) where all check-up investigations were normal and vitally stable with unremarkable ECG findings.

\section{Discussion}

Aconite tubers have been used in traditional medicine in China, Japan, Nepal, and India. Poisoning to aconite usually occurs due to either the consumption of the wild Aconitum plant or inappropriate use of these traditional/herbal medications [3]. Few reports on aconite poisoning were reported in the literature. Chan et al. reported three cases of aconite poisoning [4]. The usual presentations in these cases were hypotension, bradycardia, abdominal pain and generalized weakness. Perioral numbness, abdominal cramping and repeated vomiting were seen in only one case. The patients were treated with activated charcoal, inotropes and fluids. Another case report by Brunette et al. mentioned a case of aconite poisoning who presented with vomiting, weakness numbness, hypotension, and bidirectional ventricular tachycardia [5]. Panda et al. reported a case of overdose of Ayurvedic drug containing aconite who presented with vomiting, numbness, hypotension and bradycardia [6].

Aconitine and related alkaloids that are found in the Aconitum species are known to be highly toxic especially the roots and tubers. Aconitine is known to bind with high affinity to the open state of the voltage-sensitive sodium channels and blocking its inactivation. This results in sustained depolarization and prevents excitation of the cells. The consequence of this effect is seen in cell membranes of tissues of myocardium, nerves, and muscles, resulting in cardiotoxicity and neurotoxicity [7]. The most important effect on heart is arrhythmia, due to alteration sodium channels. This effect has been known for long; since Aconitine induced arrhythmia in animal models have been used in evaluation of anti-arrhythmic drugs [8]. It also results in activation of the ventromedial nucleus of the hypothalamus which suppresses cardiovascular system and presents as hypotension and bradycardia. The neurological effects are due alteration of so- 
dium channels which results in blocking of neurotransmission, and presented as numbness or tingling sensation [9]. Aconitine also increases the contractions of ileum due to release of acetylcholine which results in abdominal pain and diarrhea [10].

Aconite roots are rapidly absorbed from gastrointestinal tract. Hence, a short interval is observed in consumption and appearance of symptoms. This usually ranges from 10 - 20 minutes. The metabolisms of aconitine are predominantly through hydrolysis catalyzed by esterase. It also undergoes O-demethylation and other synthetic reactions which are catalyzed by cytochrome CYPP450 enzymes. The major CYP450 enzyme involved is CYP3A4 and minor CYP1A1 and CYP1A2. Our patient presented with history of appearance of symptoms one hour after the consumption of herbal remedy. He was also taking regular fluoxetine an anti-anxiety medication. Fluoxetine, a selective serotonin reuptake inhibitor is a potent inhibitor of CYP2D6, while its metabolite norfluoxetine is a moderate inhibitor of CYP3A4 and weak inhibitor of CYP1A2 [11]. It is possible that this drug interaction resulted in inhibition of metabolism of Aconitine which resulted in accumulation of the compound and thus poisoning. The metabolites thus formed are eliminated by the kidney [10].

This present case poisoning was not due to improper consumption but due to medications interaction. The patient presented with symptoms of vomiting, diarrhea, numbness and dizziness. These are some common symptoms on presentation of aconite poisoning as discussed above. Previous case reports have also mentioned arrhythmia as an important clinical feature; our patient didn't develop any arrhythmia. Though absorption of aconite occurs within 10 minutes in case of poisoning, our patient presented with symptoms after an hour. The possible reason for this could be due to inhibition of metabolism as discussed above. The management of aconite poisoning is mainly supportive depending on symptoms. There is no specific antidote. Since our patient was hypotensive, we started him on fluids. And to maintain blood pressure inotropic agent epinephrine was started. Atropine was given to counteract bradycardia. Close monitoring of vital were done. The vital kept fluctuating for up to 30 hours. After that patient slowly recovered with stable vitals, ECG also showed normal rhythm. The recovery time for patients with mild poisoning of aconite poisoning is usually 1 - 2 days [6]. Our patient similarly recovered after 30 hours, probably due to slow elimination of the metabolites overtime.

\section{Conclusion}

The use of herbal medication is quite common these days. With the common belief that these drugs are safe, patients usually tend to self-medicate. This usually leads to overdose and thus poisoning and such cases have been reported in past. Our patient represents one case where poisoning was not due to wrong consumption but due to drug-drug interaction. It is important that physicians should take drug interactions into consideration while prescribing drugs and al- 
so educate patients regarding the same. It is also important that the sale and safety of herbal medications should be properly regulated so as to ensure safety of these medications.

\section{Acknowledgements}

1). Abdulrahman Hamdi A. Assiri, Forensic Toxicologist, MSc.

2). Abdullah Alhanash, Associate Professor of Physical Chemistry, Chemistry Department, Science College, KKU.

\section{Conflicts of Interest}

The authors declare no conflicts of interest regarding the publication of this paper.

\section{References}

[1] Shaw, D., Graeme, L., Pierre, D., Elizabeth, W. and Kelvin, C. (2012) Pharmacovigilance of Herbal Medicine. Journal of Ethnopharmacology, 140, 513-518. https://doi.org/10.1016/j.jep.2012.01.051

[2] Shaw, D. (2010) Toxicological Risks of Chinese Herbs. Planta Medica, 76, 2012-2018.

[3] Chan, T.Y. (1994) Aconitine Poisoning: A Global Perspective. Veterinary and Human Toxicology, 36, 326-328. https://doi.org/10.1055/s-0030-1250533

[4] Chan, C. and Au, C. (2010) Three Cases of Aconite Root Poisoning due to Bikhama in a Hong Kong Nepalese Family. Hong Kong Journal of Emergency Medicine, 17, 158-162. https://doi.org/10.1177/102490791001700209

[5] Grimard, C., De Labriolle, A., Charbonnier, B. and Babuty, D. (2005) Bidirectional Ventricular Tachycardia Resulting from Digoxin Toxicity. Journal of Cardiovascular Electrophysiology, 16, 807-808. https://doi.org/10.1111/j.1540-8167.2005.40776.x

[6] Panda, A.K. and Debnath, S.K. (2010) Overdose Effect of Aconite Containing Ayurvedic Medicine ("MahashankhaVati”). International Journal of Ayurveda Research, 1, 183-186. https://doi.org/10.4103/0974-7788.72493

[7] Chan, T.Y. (2009) Aconite Poisoning. Clinical Toxicology, 47, 279-285. https://doi.org/10.1080/15563650902904407

[8] Sawanobori, T., Adaniya, H., Hirano, Y. and Hiraoka, M. (1996) Effects of Antiarrhythmic Agents and Mg2+ on Aconitine-Induced Arrhythmias. Japanese Heart Journal, 37, 709-718. https://doi.org/10.1536/ihj.37.709

[9] Muroi, M., Kimura, I. and Kimura, M. (1990) Blocking Effects of Hypaconitine and Aconitine on Nerve Action Potentials in Phrenic Nerve-Diaphragm Muscles of Mice. Neuropharmacology, 29, 567-572. https://doi.org/10.1016/0028-3908(90)90069-4

[10] Sato, H., Yamada, C., Konno, C., Ohizumi, Y., Endo, K. and Hikino, H. (1979) Pharmacological Actions of Aconitine Alkaloids. Tohoku Journal of Experimental Medicine, 128, 175-187. https://doi.org/10.1620/tjem.128.175

[11] Hemeryck, A. and Belpaire, F.M. (2002) Selective Serotonin Reuptake Inhibitors and Cytochrome P-450 Mediated Drug-Drug Interactions: An Update. Current Drug Metabolism, 3, 13-37. https://doi.org/10.2174/1389200023338017 